
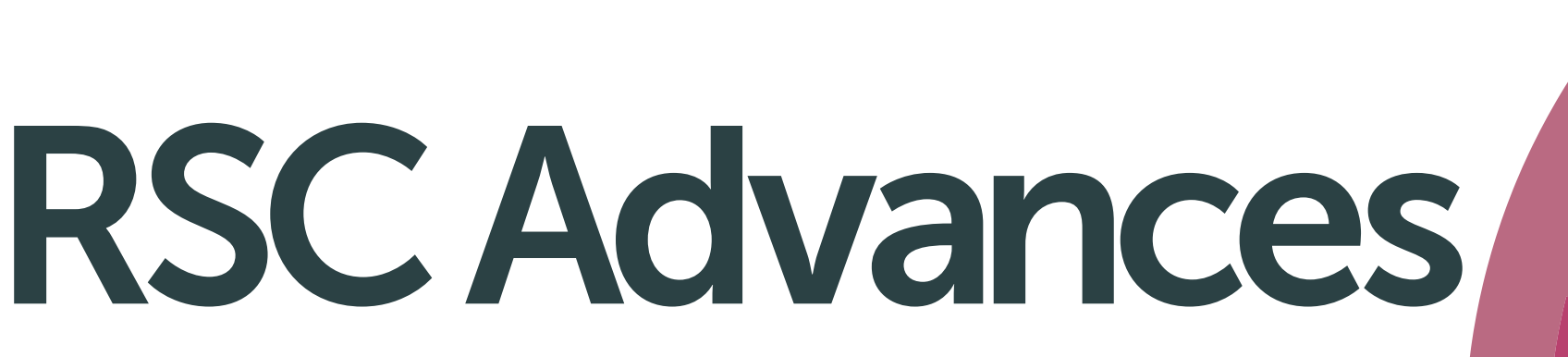

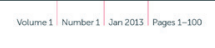

\section{RSC Advances}

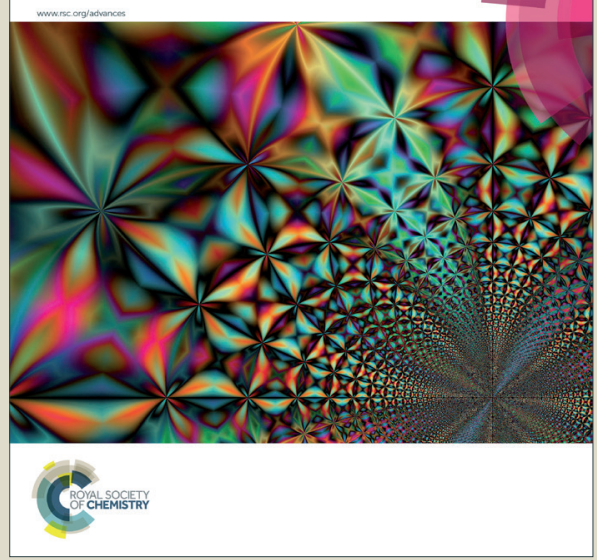

This is an Accepted Manuscript, which has been through the Royal Society of Chemistry peer review process and has been accepted for publication.

Accepted Manuscripts are published online shortly after acceptance, before technical editing, formatting and proof reading. Using this free service, authors can make their results available to the community, in citable form, before we publish the edited article. This Accepted Manuscript will be replaced by the edited, formatted and paginated article as soon as this is available.

You can find more information about Accepted Manuscripts in the Information for Authors.

Please note that technical editing may introduce minor changes to the text and/or graphics, which may alter content. The journal's standard Terms \& Conditions and the Ethical guidelines still apply. In no event shall the Royal Society of Chemistry be held responsible for any errors or omissions in this Accepted Manuscript or any consequences arising from the use of any information it contains. 


\title{
Measuring antibody coatings on gold nanoparticles by optical spectroscopy
}

\author{
Michael J. Pollitt ${ }^{*}$, Graham Buckton ${ }^{1}$, Rob Piper $^{2}$ and Steve Brocchini ${ }^{1}$ \\ ${ }^{1}$ UCL School of Pharmacy, 29-39 Brunswick Square, London, WC1N 1AX, UK \\ ${ }^{2}$ Molecular Physiology and Biophysics, University of Iowa, Iowa City, IA 52242, USA \\ *Corresponding Author: Dr M Pollitt (pollitt@talk21.com)
}

\section{Table of Contents Entry}

Coating thickness of antibody onto gold nanoparticles was estimated using changes to the absorbance spectrum. Coating was consistent with active antibody. Excess antibody used for coating nanoparticles will reduce diagnostic test sensitivity.

Keywords: gold colloid, diagnostic, antibody, coverage, spectroscopy 


\begin{abstract}
The adsorption of antibody onto gold nanoparticles to make gold-antibody conjugates is finding application in multiple areas. Gold-antibody conjugates for use in malaria diagnostics were prepared and a method of characterisation that can be applied to any gold-protein conjugate was developed. When protein adsorbs onto a gold nanoparticle, it changes the local refractive index and so changes the surface plasmon resonance of the gold particle. Changes to the surface plasmon resonance manifest in the absorbance spectrum of the conjugates. This was measured this by optical spectroscopy and relatively simple equations to convert spectral shifts to predictions of the protein layer thickness and mass coverage are presented. As with most protein adsorption reported in the literature, the results showed the protein adsorption to depend on antibody concentration, reaching a plateau at around $1 \mu \mathrm{g}$ $\mathrm{ml}^{-1}$. The coverage was estimated to be approximately $2-3 \mathrm{mg} \mathrm{m}^{-2}$ and the coating thickness estimates were approximately $10 \mathrm{~nm}$, which is consistent with active antibody. The results suggested more antibody was used in conjugate preparation than was necessary for complete coverage of the gold. This excess antibody could bind to the target antigen to reduce malaria test sensitivity. A key advantage of this characterisation method is that it is sufficiently simple to be used for quality control of conjugate production and the equations presented can be applied to other coatings on gold nanoparticles.
\end{abstract}




\section{Introduction}

Coating of materials onto gold nanoparticles find multiple applications in bio-sciences ${ }^{1,2}$. The strong colour of gold nanoparticles makes them suitable for visual observation, and is caused by the particular optical properties of gold, which was first systematically explained by $\mathrm{Mie}^{3}$. The colour is also stable; gold nanoparticles do not suffer from photobleaching, unlike organic dyes ${ }^{4}$. A particularly important application of coated gold nanoparticles is that of rapid diagnostic kits, where the colour of the gold is used by the user of the kit to observe bands indicating whether the analyte is present. These kits find particular application in the tropics, where a 'low-technology' diagnostic is useful ${ }^{5,6}$. The specific diagnostic application that motivated the work described herein was a rapid diagnostic against malaria ${ }^{7}$.

A representation of the rapid malaria diagnostic in use is shown in Figure 1. The kit detects pLDH (plasmodium lactate dehydrogenase), a protein that circulates in the blood if a patient has a malaria infection ${ }^{7}$. A small blood sample is taken from the patient by a finger prick and then mixed with the antibody-coated gold particles. This antibody binds to pLDH. The conjugate then soaks up the nitrocellulose test strip, where there is a band of a second antibody to pLDH. If the patient has malaria, the pLDH protein will bind to both antibodies and so causes a band of gold nanoparticles to be visible on the test strip.

\section{FIGURE 1}

From this a number of critical factors for the success of the diagnostic can be identified: (i) both antibodies need to be biologically active and (ii) the antibodies bound to the gold nanoparticles need to have the antigen binding sites available, namely the orientation is important. Also, if there is unbound antibody present in the conjugate solution, it will competitively bind to the pLDH malaria protein preventing binding to the gold and so reduce the sensitivity of the test kit.

Many field tests of rapid diagnostics have been conducted with differing levels of success with little definite explanation of the differences ${ }^{8}$. Also for commercialisation of the rapid diagnostics, simple quality control tests of the coated nanoparticles is essential.

The surface plasmon resonance that gives gold nanoparticles their strong colour is utilised here to measure the antibody coating thickness. Theoretical predictions show how the local refractive index environment of the particle affects its absorption spectrum. The coverage of a protein coating determines the change in local environment, which has been used here as a measurement tool. Here we measure changes in the spectrum of gold colloids with absorbed antibody, with simple laboratory equipment. This work aims to apply the physics of light absorption and scattering to the gold colloid and to present equations so this experimentally simple technique can be applied by others to the diverse range of applications of coated gold colloids.

\section{Theory}

The interaction of light with gold particles causes oscillations of the unbound valence electrons of the metal. Absorption and scattering become large close to the resonance of the electrons. Hence the phenomenon is termed surface plasmon resonance, where a plasmon is a quantised charge density wave $^{9}$. Gold colloids are coloured as absorption and scattering depends on wavelength. This is 
manifested by the complex refractive index being a strong function of the wavelength of incident light $^{10}$.

The absorbance and scattering of a particle depends on the local dielectric environment, as shown by Mulvaney ${ }^{11}$ and Mock et al. ${ }^{12}$ with solvents of different refractive indices. The local dielectric environment also changes when the particle is coated because proteins have a higher refractive index than water. The changes to absorption and scattering are manifested as changes to the wavelength of maximum absorption $\left(\lambda_{\max }\right)$ and peak absorbance $\left(A_{\max }\right)$.

Changes to $\lambda_{\max }$ were considered by various authors ${ }^{13,14}$ (see Appendix for details) by assuming the protein coated nanoparticles were spheres with a homogeneous spherical shell. The Rayleigh limit was also assumed. Comparing the case of an uncoated $50 \mathrm{~nm}$ gold particle to the exact Mie solution, the error in the wavelength of maximum absorption $\left(\lambda_{\max }\right)$ was only $0.3 \%$ but approximately $30 \%$ for $A_{\max }$. The predicted change in $\lambda_{\max }$ namely $\Delta \lambda$ is given by Equation 1 .

$\Delta \boldsymbol{\lambda}=\frac{\boldsymbol{\lambda}_{p}^{2}\left(\boldsymbol{\varepsilon}_{s}-\boldsymbol{\varepsilon}_{m}\right) g}{\boldsymbol{\lambda}_{\text {max }, 0}\left(1+2 \boldsymbol{\alpha}_{s}(1-g)\right)}$

The terms in this equation are all known: $\lambda_{p}$ is the free electron oscillation wavelength, which is $131 \mathrm{~nm}$ for gold $^{15}$; $\varepsilon$ is a dielectric constant or relative permittivity (equal to the refractive index squared) and the subscripts $s$ and $m$ refer to the shell and surrounding medium respectively; $g$ is the fraction of the total particle that is shell; $\lambda_{\max , 0}$ is the wavelength of maximum absorption for uncoated colloid and $\alpha_{s}=\left(\varepsilon_{s}-\varepsilon_{m}\right) /\left(\varepsilon_{s}+2 \varepsilon_{m}\right)$ (polarizability of a sphere of shell dielectric constant $\varepsilon_{s}$ in a medium of dielectric constant $\left.\varepsilon_{m}\right)$.

In this work it was desired to find the coating thickness from the spectral changes, so Equation 1 was inverted:

$$
g=\left(1+\alpha_{s}\right)\left(\frac{\lambda_{p}^{2}\left(\varepsilon_{s}-\varepsilon_{m}\right)}{\Delta \lambda \cdot \lambda_{\max , 0}}+2 \alpha_{s}\right)^{-1}
$$

The coating thickness $(s)$ can be calculated from the shell fraction $(g)$ and core diameter $(d)$ by geometry:

$$
s=\frac{d}{2}\left[(1-g)^{(-1 / 3)}-1\right]
$$

Equations for the change in peak absorbance $(\triangle A)$ with coating are also given in the appendix. The mass of protein absorbed per unit area (coverage, $\Gamma$ ) is of interest as well as the coating thickness. This can be calculated using de Feijter's formula ${ }^{16}$ :

$$
\Gamma=s \frac{n_{s}-n_{m}}{d n / d c}
$$

Where, $n$ is the refractive index (for the shell $\left(n_{s}\right)$ and the surrounding medium $\left(n_{m}\right)$, which in this system is essentially water, which has a refractive index of 1.335 at $550 \mathrm{~nm}$ ) and $d n / d c$ is the refractive index increment (typically $0.19 \mathrm{ml} / \mathrm{g}$ for $\operatorname{protein}^{17}$ )

A key advantage of Equation 2 is that it is sufficiently simple to be solved for a given set of spectral data on a spreadsheet. However, the refractive index of the shell, in this case the adsorbed 
antibody layer needs to be estimated. The refractive index of the protein coating is lower than that of dry protein due to entrapped water within the layer ${ }^{18}$. Dry protein has a refractive index in the region of 1.5 to $1.6^{18-20}$. Zhou et al. ${ }^{21}$ assumed a refractive index for pure protein between 1.450 and 1.465. For an IgG layer (on a hydrophobic surface), Lassen and Malmsten ${ }^{22}$ used ellipsometry to show that the layer refractive index to vary with coverage (1.34 to 1.39), consistent with a decreasing fraction of water in the layer. Estimates of refractive index ranged from 1.40 to 1.44 for $\operatorname{IgG}$ on gold nanoparticles, using a combination of spectral changes and dynamic light scattering (DLS), nanoparticle tracking analysis (NTA) and differential centrifugation sedimentation (DCS $)^{23}$. A combination of optical waveguide lightmode spectroscopy (OWLS) and a quartz crystal microbalance (QCM) was used by Vörös ${ }^{24}$ to measure IgG coverage and adsorbed mass including water respectively. From these the density of the layer and so its refractive index was found as 1.38 on poly(tetrafluoroethylene) and 1.42 on $\mathrm{TiO}_{2}$.

Equation 3 is plotted in Figure 2A, for different possible shell refractive indices, and shows the impact of this uncertainty in refractive index: a shell of a given thickness causes more peak shift $(\Delta \lambda)$ if it has a higher refractive index. The calculated coverage $(\Gamma)$, shown in Figure $2 \mathrm{~B}$, is much less sensitive to the value of protein refractive index $\left(n_{s}\right)$ used than the calculated layer thickness $(s)$. This is because the terms with strong $n_{s}$ dependence: $\left(n_{s}-n_{m}\right)$ in Equation 4 and $\left(\varepsilon_{s}-\varepsilon_{m}\right)$ in Equation 3 partially cancel. In Figure $2 \mathrm{C}$, these data were re-plotted to directly observe the effect of shell refractive index on the shell thickness from an example set of spectral data. From Figure $2 \mathrm{C}$ it is notable that the layer thickness curves calculated from $\Delta \lambda$ and $\Delta A$ are roughly parallel. If they intersected cleanly, the combination of $\Delta \lambda$ and $\Delta A$ could be used to find both the coating thickness and refractive index. However, roughly the same information is gained from both $\Delta \lambda$ and $\Delta A$, so $n_{s}$ is required to find $s$. The lower sensitivity to shell refractive index in the calculation of coverage can also be observed.

\section{FIGURE 2}

\section{Materials and Methods}

Colloidal gold was prepared by a modification to the Frens ${ }^{25}$ method. All glassware was first cleaned using aqua regia, to remove any initial traces of gold colloid that would serve as nucleation centres, rinsed with deionised water and oven dried. Deionised water $(100 \mathrm{ml})$ and $\mathrm{HAuCl}_{4} \cdot 3 \mathrm{H}_{2} \mathrm{O}\left(10 \mathrm{mg} \mathrm{ml} l^{-1}\right.$, $1.0 \mathrm{ml}$, Sigma) were mixed and boiled under reflux with constant stirring. $\mathrm{Na}_{3}$-citrate $\bullet 2 \mathrm{H}_{2} \mathrm{O}(10 \mathrm{mg}$ $\mathrm{ml}^{-1}, 12.0 \mathrm{ml}$, Sigma) was added and boiled for $2 \mathrm{~min}$. The solution was pale pink at the end of this step (nucleation). $\mathrm{HAuCl}_{4} \cdot 3 \mathrm{H}_{2} \mathrm{O}\left(10 \mathrm{mg} \mathrm{ml}^{-1}, 8.4 \mathrm{ml}\right)$ was added and the solution turned black after approximately $10 \mathrm{~s}$ and then opaque purple after a further $10 \mathrm{~s}$. Boiling was continued for 2 min. All reagent solutions were filtered $(0.22 \mu \mathrm{m}$, Millipore Express PES) prior to addition.

The particle size of the gold colloid was measured by Transmission Electron Microscopy (FEI-Philips, Eindhoven, Netherlands, BioTwin CM120 with a Lab 6 emitter and $120 \mathrm{kV}$ accelerating potential), as $52.6 \mathrm{~nm}$ (number mean) with a standard deviation of $10.0 \mathrm{~nm}$. The particles were roughly spherical and approximately normally distributed. The size measurement was repeated one year after preparation and no significant difference in size was found, indicating a stable colloid. 
The concentration of the colloid was found by assuming complete reduction of the $\mathrm{HAuCl}_{4}{ }^{25-}$ 26. The mass concentration of gold in all batches was $387 \mu \mathrm{g} \mathrm{ml}^{-1}$, volume concentration $2.01 \times 10^{-5} \mathrm{~m}^{3} \mathrm{~m}^{-3}(20 \mathrm{ppmv})$ and for $50 \mathrm{~nm}$ diameter particles, a molar particle concentration of $0.5 \mathrm{nM}$.

Conjugates were prepared at various concentrations of antibody. Antibody (type 6C9, a kind gift of Mike Makler of Flow Inc., $40 \mu \mathrm{l}, 1.6$ to $200 \mu \mathrm{g} \mathrm{ml}^{-1}$ ) was mixed with Tris-HCl buffer (40 $\mu 1,0.1$ $\mathrm{M}, \mathrm{pH}$ 8.4) and gold colloid $(200 \mu \mathrm{l})$. Colloid only controls were prepared in the same manner, but with the antibody replaced by buffer. The samples were diluted ten fold and the visible spectrum was measured using a Cary 3E spectrophotometer (Varian, Palo Alto, CA) with $0.2 \mathrm{~nm}$ spectral bandwidth, $0.1 \mathrm{~nm}$ resolution and $0.1 \mathrm{~s}$ (3 chopper cycles) averaging per point. On this instrument, the monochromator is a diffraction grating and the detector is a photomultiplier tube. Repeat measurements included repeating the antibody conjugation step rather than just the spectroscopy. The peak absorbance $\left(\lambda_{\max }\right)$ was found by fitting a quadratic equation (least squares method) to the absorbance from 530 to $565 \mathrm{~nm}$ and the maximum found by differentiation. This was found to result in a lower standard deviation in $\lambda_{\max }$ than by using the peak of the raw data because the fitting smooths noise from the spectrum.

\section{Results and Discussion}

An example of the raw optical density data for both uncoated gold colloid and conjugates is shown in Figure 3. A peak at approximately $550 \mathrm{~nm}$ is observed, which gives the colloid used for this work its distinctive purple colour. Note that the absorbance spectrum of gold colloid depends on particle size, so smaller, more common gold colloids have a red colour. For the purposes of a rapid diagnostic, larger gold is advantageous because the colour is more intense per surface area, so maximising the sensitivity. The red shift in the peak absorbance wavelength on conjugation $\left(\lambda_{\max }\right.$ increase) is difficult to see by eye, but can be observed by the quadratic fitting method in Figure 4.

\section{FIGURES 3-4}

If the red-shift was caused by coagulation of the colloid (rather than antibody conjugation to the gold) then the peak absorbance would fall and would cause an increase in absorbance at approximately $750 \mathrm{~nm}$ from the absorbance of the aggregates ${ }^{27}$. However, as Figure 3 shows the absorbance of the conjugates between 700 and $800 \mathrm{~nm}$ is slightly lower than for the uncoated colloid and Figure 3 shows an increase in $A_{\max }$ on conjugation, so coagulation as the cause of red-shift can be discounted.

The data in Figure 3 were used with Equation 4 to calculate the antibody coverage on the gold at the different bulk antibody concentrations, with results shown in Figure 5. Small changes in the medium refractive index $\left(n_{m}\right)$ from buffer solute and unadsorbed protein have a negligible effect on $\lambda_{\max }$ (check via Equation A10) and contributions from adsorbed citrate (residue from the colloid preparation) are negligible due to the small size of the citrate molecule.

The measurements of coverage (Figure 5) are broadly consistent with the variety of measurements of antibody adsorption reported in the literature ${ }^{21,28-31}$. Figure 5 shows that at the low concentrations, the estimated coverage is larger than that predicted from assuming all of the antibody is 
adsorbed onto the theoretical surface area of the gold colloid. Changes to the layer refractive index $\left(n_{s}\right)$ can not eliminate this observation.

\section{FIGURE 5}

The five lowest concentrations in Figure 5 correspond to between 4 and 65 antibody molecules per gold particle. To put this in perspective, a coverage of $2.6 \mathrm{mg} \mathrm{m}^{-2}$ for end-on orientation $^{31}$ corresponds to approximately 80 antibody molecules on a $50 \mathrm{~nm}$ particle. Therefore, for the concentrations studied in Figure 5, it is likely that only parts of the surface will be covered.

It is postulated that a gold particle with isolated adsorbed antibody molecules has the optical properties as if there was a shell of molecules and so the coverage is overestimated by Equation 4. Rather than using Equations 1-4, it is possible (but more complicated) to model the coated spheres using the Mie model and the heterogeneous coating as a series of many homogeneous layers ${ }^{32-33}$. However, the more complicated models seems to have little merit when the unknown refractive index of the layer is so significant in the layer thickness calculation (see Figure 2). No models considering partial coatings have been found in the literature.

Figure 5 also shows that at higher antibody concentrations, the coverage is less than predicted from complete adsorption, implying there is unadsorbed antibody. The higher concentrations were investigated because they are needed to prevent sodium chloride agglutination of the nanoparticles, which is the traditional method of determining the appropriate amount of antibody to $\operatorname{add}^{34}$. From the perspective of the diagnostic kit, these concentrations need to be avoided: (1) the un-adsorbed antibody will competitively or preferentially interact with the test protein thus reducing the sensitivity of the test and (2) if more antibody is used than needed for complete coverage, this increases the cost of the kit.

The conformation of the adsorbed antibody is critical in determining whether it is biologically active. Ideally, the Fc region would be absorbed to the surface and the antigen binding Fab regions are free to bind to the antigen rather than also being adsorbed to the surface. Therefore, the 'end-on' conformation is desired over the 'side-on' conformation ${ }^{35}$. An active antibody molecule is a ' $Y^{\prime}$ shape and the size has been estimated by many authors, with the long length as approximately $12-14 \mathrm{~nm}^{21,36}$, so an active antibody coating ('end-on' conformation) should have a thickness of approximately $12 \mathrm{~nm}$. Thinner coatings of around $6 \mathrm{~nm}$ would correspond to the 'side-on' conformation. The layer thickness was calculated from the peak shifts using Equations 2 and 3 and results are shown in Figure 6. They show the shell thickness $(s)$ of approximately $10 \mathrm{~nm}$ and independent of antibody concentration, above approximately $1 \mu \mathrm{g} \mathrm{ml}^{-1}$. This nearly corresponds to end-on antibody size $(12 \mathrm{~nm})$. However, if the layer refractive index $\left(n_{s}\right)$ is 1.40 rather than 1.38 then the coating thickness is approximately $6 \mathrm{~nm}$, which is closer to the side-on orientation. Also, the layer refractive index $\left(n_{s}\right)$ depends on the water content of the layer ${ }^{18,23}$ which is not necessarily constant with antibody concentration. However if $n_{s}$ changes, it is likely to increase with protein concentration; that is, because of less water in the protein layer. This would correspond to thinner layers at higher concentrations (Figure 2A), which seems unlikely physically. Therefore, a key problem with the method is the sensitivity to layer refractive index. However for antibodies, a refractive index of 1.38 appears to be a more accurate estimate than 1.40 for the refractive index. Hence this method for determining coating thickness for antibody coating potentially has wide application. 


\section{FIGURE 6}

As proteins are anisotropic, the refractive index will depend on the orientation (birefringence, a feature exploited in circular dichroism spectroscopy). Therefore the protein layer refractive index will depend on the orientation of the molecules. However, the magnitude of the birefringence is unknown and was not measured by Lassen and Malmsten ${ }^{22}$ or by Vörös ${ }^{24}$. The birefringence is assumed to be negligible compared to the changes in refractive index from the water content of the protein layer.

There have been other measurements of antibody film thicknesses, such as 3-7 nm (depending on concentration) on gold by quartz crystal microbalance and surface acoustic wave and $6 \mathrm{~nm}$ by $\mathrm{AFM}^{21}$; a 2-2.5 nm layer thickness on mica measured by $\mathrm{AFM}^{37}$ and $4 \mathrm{~nm}$ on silica by neutron reflection $^{29}$. Comparisons are difficult because of the $n_{s}$ uncertainty for the results presented here and that other measurements have been made on flat surfaces rather than particles. Bell et al. ${ }^{23}$ used a wide range of antibody concentrations $(0.1 \mu \mathrm{g} / \mathrm{ml}$ to $1 \mathrm{mg} / \mathrm{ml})$ and measured a range of thicknesses using DLS, NTA and DCS up to approximately $12 \mathrm{~nm}$.

This technique could also be applied to other metallic particles that have a plasmon resonance, such as silver. The equations for absorbance changes on coating should still apply, but refractive index data for the other metal would need to be used. Non-metallic particles typically do not have an absorbance maximum, so the only optical changes on absorption would be an increase in scattering from the larger particle size. Analytically this would be difficult to exploit as a low wavelength would have to be used and this is sensitive to dust. Also, the equation derived for the peak shift (Equation 1) does not apply because the dielectric constant of non-metallic particles does not follow the Drude model (Equation A8) ${ }^{9}$.

\section{Conclusion}

This method gives an estimate of the antibody coverage in a non-invasive way and has shown that a layer is definitely formed on the gold colloid. The main difficulty with the method is the sensitivity of the result to the protein layer refractive index, which must be determined by other means and possible problems when the coverage is so low that the adsorbed antibody molecules are isolated from each other. For antibodies, the best estimate of the refractive index is 1.38. Absorbance peak shifts have been used before to consider protein binding to gold colloids ${ }^{14,18,32}$ and antigen binding to adsorbed antibodies $^{9,38,39}$. However, this work represents the first to show the sensitivity of the thickness measurement to protein layer refractive index and that coverage can also be calculated. The coverage measurement is less sensitive to protein refractive index.

Considering implications for the diagnostic kit, the most likely conformation of the protein is end-on, which should retain antibody activity. The derived protein coverage shows excess antibody was used, which reduces the sensitivity of the rapid diagnostic. The method is sufficiently simple to be used for quality control of antibody conjugate production and the equations presented can be applied to other coatings on gold nanoparticles. 


\section{Acknowledgements}

The authors would like to thank the UCL School of Pharmacy for a PhD studentship for MJP and Mike Makler of Flow Inc. for the gift of the antibody. SB is grateful for funding from the UK Engineering \& Physical Sciences Research Council (EPSRC) for the EPSRC Centre for Innovative Manufacturing in Emergent Macromolecular Therapies. Financial support from the consortium of industrial and governmental users for the EPSRC Centre is also acknowledged. SB is also grateful for funding from NIHR Biomedical Research Centre at Moorfields Hospital and the UCL Institute of Ophthalmology, Moorfields Special Trustees, the Helen Hamlyn Trust (in memory of Paul Hamlyn), Fight for Sight and Freemasons Grand Charity.

Financial Interest Statement: The authors declare no competing financial interest

\section{$5 \quad$ Theory Appendix}

This appendix considers the derivation of Equation 1 (variation in the wavelength of peak absorption, $\lambda_{\max }$, with coating thickness) and the equivalent equation for the peak absorption, $A_{\max }$.

The absorbance of any monodisperse colloid is given by Equation A1, where $A$ is the absorbance, $N$ is the number of particles and $l$ is the path length. $C_{e x t}$ is the extinction cross section, that is the equivalent area blocked by each particle ${ }^{11}$.

$A=\frac{N C_{e x t} l}{2.303}$

Considering the relative change in absorbance $(\triangle A / A)$ allows factors such as concentration to cancel as this remained constant. Equation A2 is obtained, where the subscript 0 refers to uncoated particles:

$$
\frac{A_{\text {conjugate }}-A_{0}}{A_{0}}=\frac{\Delta A}{A}=\frac{C_{\text {ext }, \text { conjugate }}}{C_{\text {ext }, 0}}-1
$$

$C_{e x t}$ is the sum of two components, absorption $\left(C_{a b s}\right)$ and scattering $\left(C_{s c a}\right)$, as given by Equations A3 to A $5^{9,15,40}$

$C_{a b s}=\frac{\pi^{2}(d+2 s)^{3} n_{\text {solvent }} \operatorname{Im}(F(\boldsymbol{\varepsilon}))}{\lambda}$

$C_{\text {sca }}=\frac{2 \pi}{3}\left(\frac{\pi n_{\text {solvent }}}{\lambda}\right)^{4}(d+2 s)^{6}|F(\boldsymbol{\varepsilon})|^{2}$

$F(\boldsymbol{\varepsilon})=\frac{\left(\boldsymbol{\varepsilon}_{s}-\boldsymbol{\varepsilon}_{m}\right)\left(\boldsymbol{\varepsilon}_{c}+2 \boldsymbol{\varepsilon}_{s}\right)+f\left(\boldsymbol{\varepsilon}_{c}-\boldsymbol{\varepsilon}_{s}\right)\left(\boldsymbol{\varepsilon}_{m}+2 \boldsymbol{\varepsilon}_{s}\right)}{\left(\boldsymbol{\varepsilon}_{s}+2 \boldsymbol{\varepsilon}_{m}\right)\left(\boldsymbol{\varepsilon}_{c}+2 \boldsymbol{\varepsilon}_{s}\right)+2 f\left(\boldsymbol{\varepsilon}_{s}-\boldsymbol{\varepsilon}_{m}\right)\left(\boldsymbol{\varepsilon}_{c}-\boldsymbol{\varepsilon}_{s}\right)}$

Other authors ${ }^{11,13,15}$ do not consider the scattering term as it is small in the Rayleigh limit, which is valid for small gold particles. As $50 \mathrm{~nm}$ diameter gold particles are at the borderline or outside the Rayleigh limit the scattering term was included and contributes approximately $20 \%$ of the 
extinction. Hence optical density is a more accurate term for the extinction measured on a spectrometer but the symbol $A$ is still used.

To find $\lambda_{\max }$ the expression for the extinction coefficient $\left(C_{e x t}\right)$ is maximised by setting the denominator of Equation A5 to zero (as shown in Equation A6) and solving for $\varepsilon_{s}$, the dielectric constant of the core, which for gold is a strong function of wavelength.

$$
\begin{aligned}
& \left(\boldsymbol{\varepsilon}_{s}+2 \boldsymbol{\varepsilon}_{m}\right)\left(\boldsymbol{\varepsilon}_{c}+2 \boldsymbol{\varepsilon}_{s}\right)+2 f\left(\boldsymbol{\varepsilon}_{s}-\boldsymbol{\varepsilon}_{m}\right)\left(\boldsymbol{\varepsilon}_{c}-\boldsymbol{\varepsilon}_{s}\right)=0 \\
& \boldsymbol{\varepsilon}_{c}=-2 \boldsymbol{\varepsilon}_{s} \frac{\boldsymbol{\varepsilon}_{s}(1-f)+\boldsymbol{\varepsilon}_{m}(2+f)}{\boldsymbol{\varepsilon}_{s}(1+2 f)+2 \boldsymbol{\varepsilon}_{m}(1-f)}=-2 \boldsymbol{\varepsilon}_{s} \frac{1-f \boldsymbol{\alpha}_{s}}{1+2 f \boldsymbol{\alpha}_{s}}
\end{aligned}
$$

To relate the dielectric constant $\left(\varepsilon_{c}\right)$ to $\lambda_{\max }$, the Drude dielectric function is used. This function models the free electrons in the conductor (gold) as a simple harmonic oscillator about the nuclei. As the shell and medium are non-absorbing, Equation A7 refers to real part of the dielectric constant $\left(\varepsilon^{\prime}\right)$ the expression for which, by the Drude model, is given by Equation A8. The free electron oscillation wavelength is $\lambda_{p}$ (dependent on the free electron density), $c$ is the speed of light (in vacuum) and $\gamma$ is a damping factor ${ }^{9}$.

$$
\boldsymbol{\varepsilon}^{\prime}=1-\frac{\left(2 \pi c / \lambda_{p}\right)^{2}}{(2 \pi c / \lambda)^{2}+\gamma^{2}}
$$

An approximation to this function is used given by Equation A9 where $\varepsilon_{\infty}$ is the interband contribution to the dielectric function ${ }^{13}$ :

$$
\boldsymbol{\varepsilon}^{\prime}=\boldsymbol{\varepsilon}_{\infty}-\frac{\lambda^{2}}{\lambda_{p}^{2}}
$$

This approximation for $\varepsilon$ was also compared to experimental values of $\varepsilon^{\prime}$ for gold and found to be a reasonable match around $\lambda_{\max }$. Note that the approximation used by Bohren and Huffman is not a good approximation for gold at those wavelengths.

By considering the difference in dielectric constant between coated spheres (Equation A7) and uncoated spheres $\left(\varepsilon_{c}=-2 \varepsilon_{m}\right.$.), Equation A10 is derived, where $f$ is the fraction of the total particle that is core:

$\boldsymbol{\varepsilon}_{c}+2 \boldsymbol{\varepsilon}_{m}=\frac{\lambda_{\max }^{2}}{\lambda_{p}^{2}}-\frac{\lambda_{\max , 0}^{2}}{\lambda_{p}^{2}}=-2 \boldsymbol{\varepsilon}_{s} \frac{\boldsymbol{\varepsilon}_{s}(1-f)+\boldsymbol{\varepsilon}_{m}(2+f)}{\boldsymbol{\varepsilon}_{s}(1+2 f)+2 \boldsymbol{\varepsilon}_{m}(1-f)}+2 \boldsymbol{\varepsilon}_{m}$

By substituting $g=1-f, \lambda_{\max }=\lambda_{\max , 0}+\Delta \lambda$ noting that $\lambda_{\max } \approx \lambda_{\max , 0}$ so $\lambda^{2}-\lambda_{\max , 0}{ }^{2} \approx 2 \lambda_{\max , 0} \Delta \lambda$ and rearranging:

$\Delta \boldsymbol{\lambda}=\frac{\boldsymbol{\lambda}_{p}^{2}\left(\boldsymbol{\varepsilon}_{s}-\boldsymbol{\varepsilon}_{m}\right) g}{\boldsymbol{\lambda}_{\text {max }, 0}\left(1+2 \boldsymbol{\alpha}_{s}(1-g)\right)}$

The peak absorbance $\left(A_{\max }\right)$ is found by substituting the value of $\varepsilon_{c}$ at $\lambda_{\max }$ into Equations A3 to A5. 
Graphical abstract for the table of contents

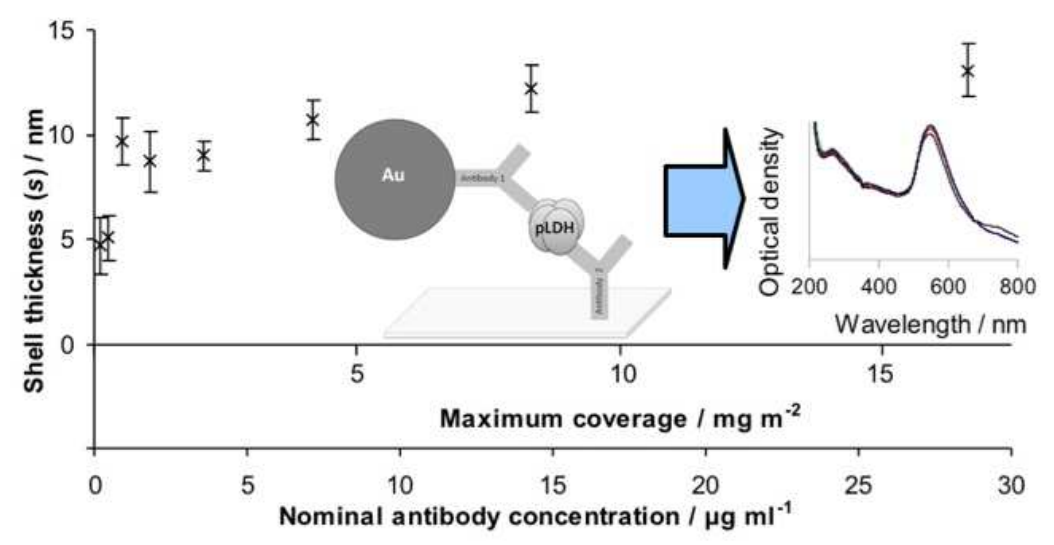

Coating thickness estimates of coated gold nanoparticles was achieved to avoid reduction of diagnostic sensitivity from excess antibody.

\section{Figure 1}

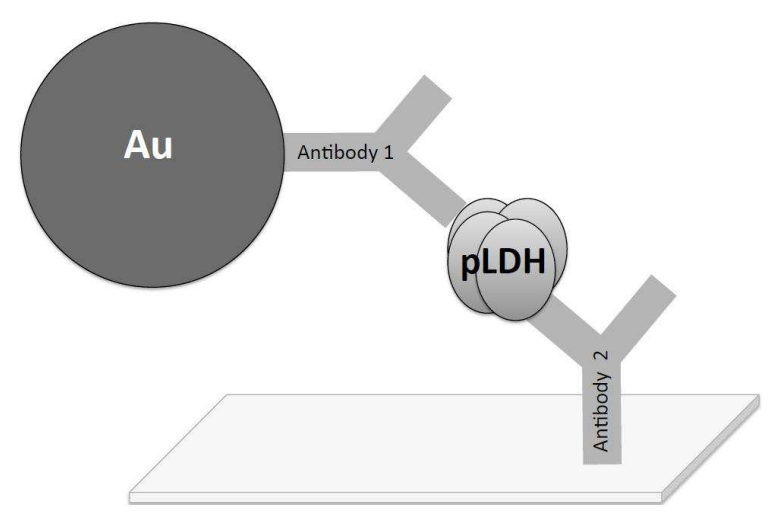

Figure 1. Ilustration of the malaria rapid diagnostic with bound malaria protein (pLHD) linking the gold to a localised region on the nitrocellulose test strip. 
Figure 2
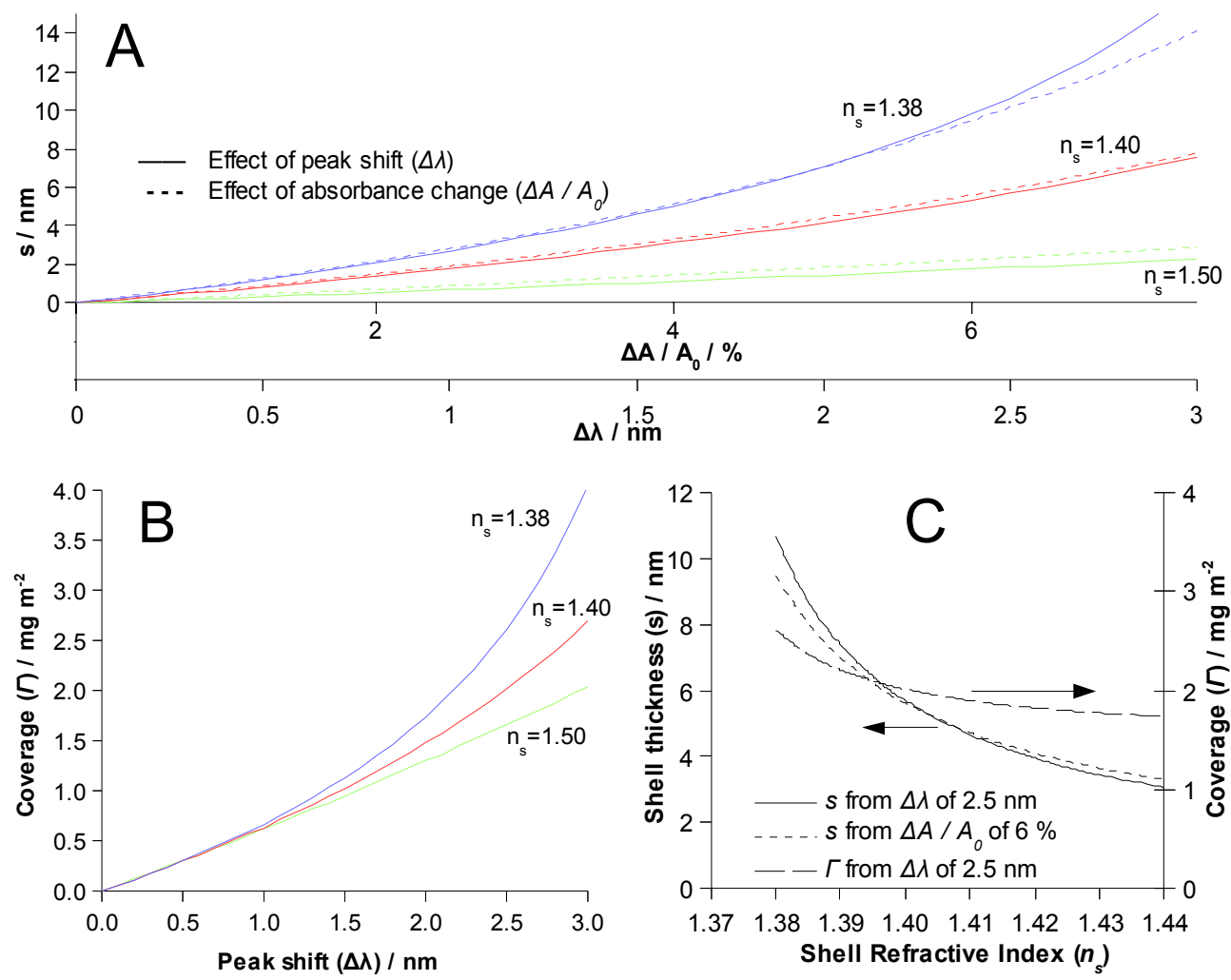

Figure 2. A: Predictions of coating thickness $(s)$ from spectral changes. B: Predictions of coverage $(\Gamma)$. C: Impact of shell refractive index $\left(n_{s}\right)$ on the predictions. All predictions are for $50 \mathrm{~nm}$ gold particles. 
Figure 3

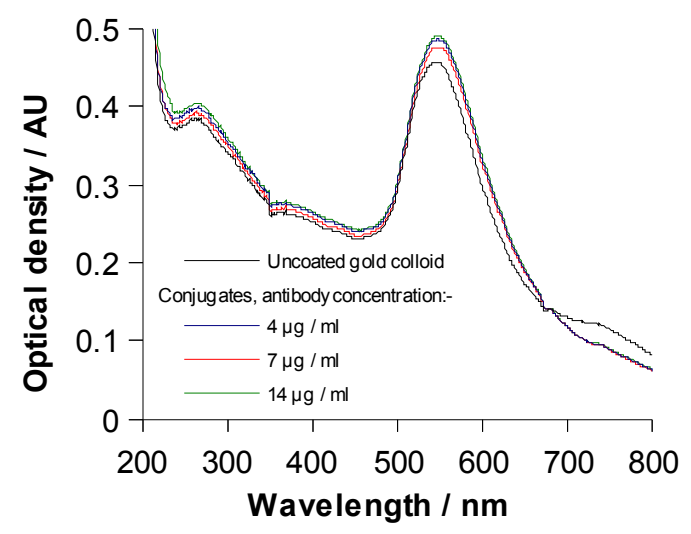

Figure 3. Changes to the UV-visible extinction spectrum on conjugation. All samples were ten fold diluted prior to measurement. These spectra only were measured with $1 \mathrm{~nm}$ resolution and $2 \mathrm{~nm}$ spectral bandwidth. Note that the spectrometer has a source change at $350 \mathrm{~nm}$. 
Figure 4

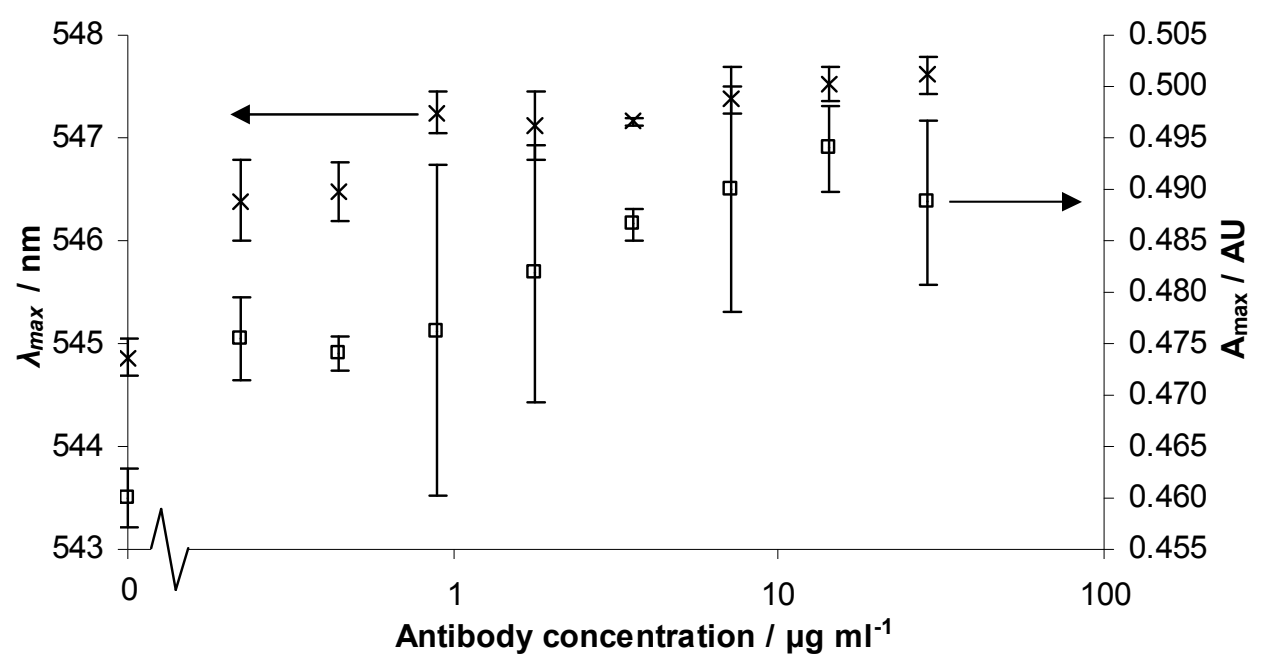

Figure 4. Variation in conjugate peak absorbance with antibody concentration. Wavelength $\left(\times, \lambda_{\max }\right)$, Optical density $\left(\square, A_{\max }\right)$. Mean $\pm \mathrm{SD}, \mathrm{n}=3$ preparations. 
Figure 5

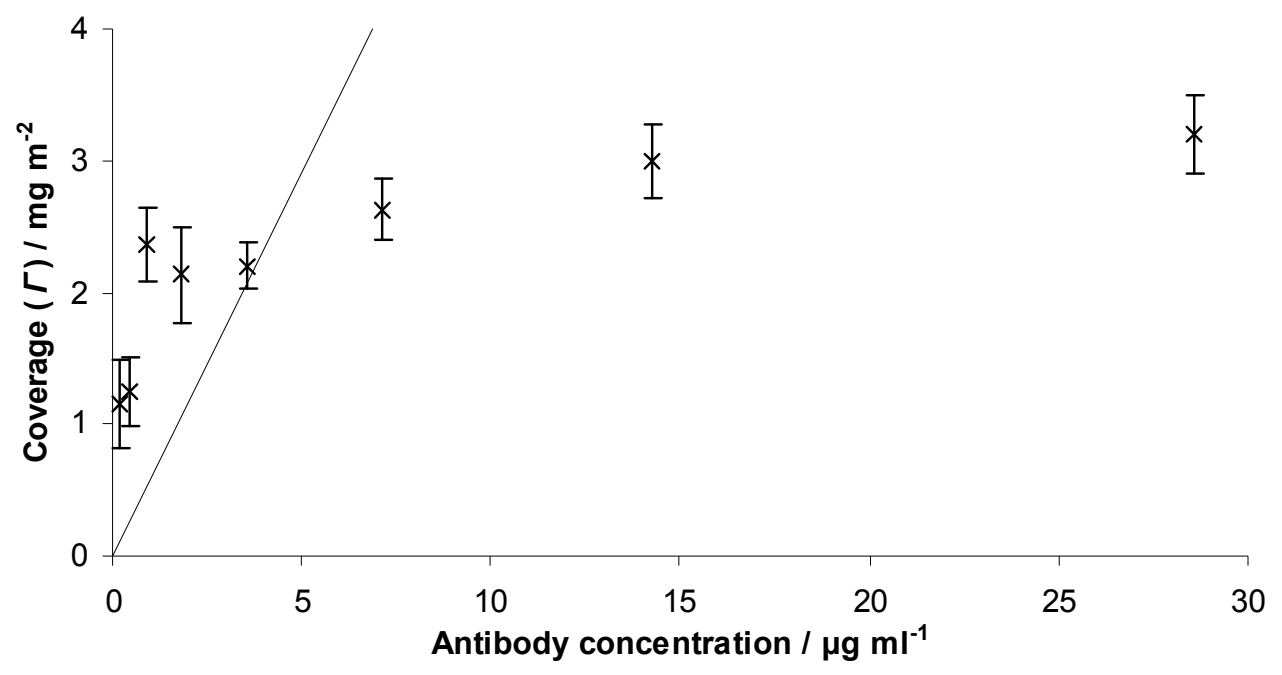

Figure 5. Variations in antibody coverage on gold colloid with antibody concentration $(\times)$ found from changes in $\lambda_{\max }$ and assuming $n_{s}=1.38$. The line represents the coverage if all antibody is adsorbed. Mean $\pm \mathrm{SD}, \mathrm{n}=3$, error bars do not include the uncertainty in coating refractive index. 
Figure 6

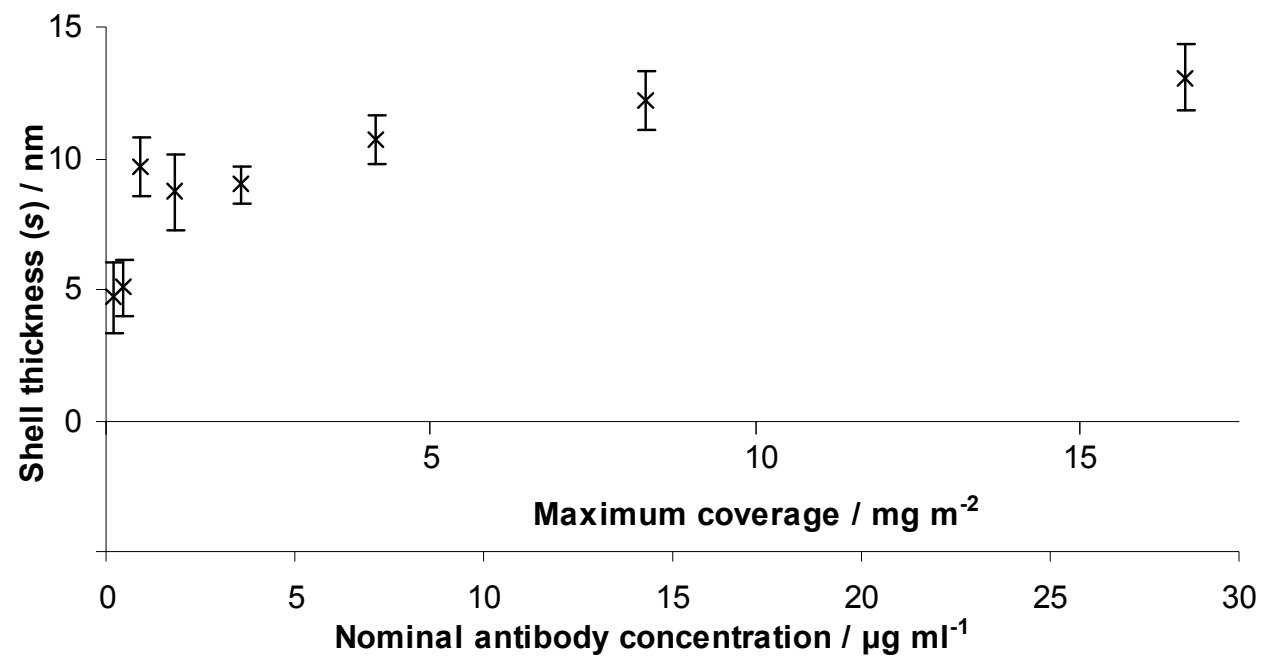

Figure 6. Antibody coating thickness on gold colloid found from changes in $\lambda_{\max }$ and assuming a coating refractive index $\left(n_{s}\right)$ of 1.38. Mean $\pm \mathrm{SD}, \mathrm{n}=3$, error bars do not include the uncertainty in coating refractive index. 
1. J. Cao, T. Sun, K.T.V. Grattan, Sensor. Actuat. B: Chem, 2014, 195, 332-351.

2. S. Rana, A. Bajaj, R. Mout, V.M. Rotello, Adv. Drug Del. Rev., 2012, 64, 200-216.

3. G. Mie, Annaler Der Physik, 1908, 25, 377-445.

4. K. Aslan, P. Holley, L. Davies, J.R. Lakowicz, C.D. Geddes, J. Am. Chem. Soc., 2005, 127, 12115-12121.

5. F. Chappuis, E. Alirol, V. d'Acremont, E. Bottieau, C.P. Yansouni, Clin. Microbiol. Infect., 2013, 19, 422-31.

6. J.C. Mouatcho, J.P. Goldring, J. Med. Microbiol., 2013, 62, 1491-505.

7. R. Piper, J. Lebras, L. Wentworth, A. Hunt-Cooke, S. Houzé, P. Chiodini, M. Makler, Am. J. Trop. Med. Hyg., 1999, 60, 109-118.

8. A.H. Moody, Clin.Microbiol. Rev., 2002, 15, 66-78.

9. C.F. Bohren, D.R. Huffman, Absorption and scattering of light by small particles, John Wiley \& Sons Inc., New York, 1983.

10. M.J. Weber (Ed.), CRC Handbook of Laser Science and Technology Volume 4. Optical Materials: Part 2, CRC Press Inc., Boca Raton, FL, 1986.

11. P. Mulvaney, Langmuir, 1996, 12, 788-800.

12. J.J. Mock, D.R. Smith, S. Schultz, Nano Lett., 2003, 3, 485-491.

13. N.G. Khlebtsov. J. Quant. Spect. Radiative Transfer, 2004, 89, 143-153.

14. D. Eck, C.A. Helm, N.J. Wagner, K.A. Vaynberg, Langmuir, 2001, 17, 957-960.

15. A.C. Templeton, J.J. Pietron, R.W. Murray, P. Mulvaney, J. Phys. Chem. B, 2000, 104, 564570.

16. J.A. De Feijter, J. Benjamins, F.A. Veer, Biopolymers, 1978, 17, 1759-1772.

17. H. Zhao, P.H. Brown, P. Schuck, Biophys. J., 2011, 100, 2309-2317.

18. N.G. Khlebtsov, V.A. Bogatyrev, L.A. Dykman, A.G. Melnikov, J. Colloid Interface Sci., 1996, 180, 436-445.

19. A.A. Fisk, Proc. Natl. Acad. Sci. U. S. A., 1950, 36, 518-523.

20. J. Benesch, A. Askendal, P. Tengvall, J. Colloid Interface Sci., 2002, 249, 84-90.

21. C. Zhou, J.-M Friedt, A. Angelova, K.-H. Choi, W. Laureyn, F. Frederix, L.A. Francis, A. Campitelli, Y. Engelborghs, G. Borghs, Langmuir, 2004, 20, 5870-5878.

22. B. Lassen, M. Malmsten, J. Colloid Interface Sci., 1996, 180, 339-349.

23. N.C. Bell, C. Minelli, A.G. Shard, Anal. Methods, 2013, 5, 4591-4601.

24. J. Vörös, Biophys. J., 2004, 87, 553-561.

25. G. Frens, Nat. Phys. Sci., 1973, 241, 20-22.

26. M.A. Hayat, (Ed.) Colloidal gold: principles, methods and applications, Academic Press, San Diego, California, 1989.

27. H.M. Lindsay, M.Y. Lin, D.A. Weitz, P. Sheng, Z. Chen, R. Klein, P. Meakin, Faraday Discussions, 1987, 83, 153-165.

28. K. Kaur, J.A. Forrest, Langmuir, 2012, 28, 2736-2744.

29. H. Xu, J.R. Lu, D.E. Williams, J. Phys. Chem. B, 2006, 110, 1907-1914.

30. M.G.E.G. Bremer, J. Duval, W. Norde, J. Lyklema, Colloid Surf. A-Physicochem. Eng. Asp., 2004, 250, 29-42.

31. J. Buijs, J.W.Th. Lichtenbelt, W. Norde, J. Lyklema, Colloid Surf. B-Biointerfaces, 1995, 5, 11-23.

32. N.G. Khlebtsov, V.A. Bogatyrev, B.N. Khlebtsov, L.A. Dykman, P. Englebienne, Colloid J., 2003, 65, 622-635.

33. O.B. Toon, T.P. Ackerman, Appl. Optics, 1981, 20, 3657-3660.

34. W.P. Faulk, G.M. Taylor, Immunochemistry, 1971, 8, 1081-1083.

35. J. Buijs, D.D. White, W. Norde, Colloid Surf. B-Biointerfaces, 1997, 8, 239-249.

36. B. Carrasco, J. Garcia de la Torre, K.G. Davis, S. Jones, D. Athwal, C. Walters, D.R. Burton, S.E. Harding, Biophysical Chemistry, 2001, 93, 181-196.

37. M. Bergkvist, J. Carlsson, T. Karlsson, S. Oscarsson, J. Colloid Interface Sci., 1998, 206, $475-$ 481.

38. P. Englebienne, Analyst, 1998, 123, 1599-1603.

39. P. Englebienne, A. Van Hoonacker, M. Verhas, Analyst, 2001, 126, 1645-1651.

40. A. Kokhanovsky, Optics of light scattering media: problems and solutions, John Wiley \& Sons Ltd, 1999. 


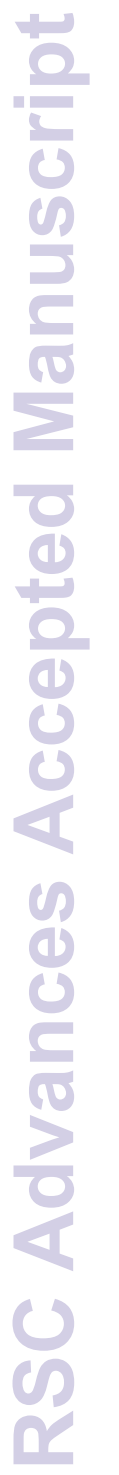

